Vision and Image Understanding

Elsevier Editorial System(tm) for Computer

Manuscript Draft

Manuscript Number: CVIU-17-1R1

Title: Polarisation Photometric Stereo

Article Type: Research paper

Keywords: Polarisation; photometric stereo; 3D reconstruction

Corresponding Author: Dr. Gary Atkinson, PhD

Corresponding Author's Institution: University of the West of England

First Author: Gary Atkinson, PhD

Order of Authors: Gary Atkinson, PhD

Abstract: This paper concerns a novel approach to fuse two-source photometric stereo (PS) data with polarisation information for complete surface normal recovery for smooth or slightly rough surfaces. PS is a well-established method but is limited in application by its need for three or more well-spaced and known illumination sources and Lambertian reflectance. Polarisation methods are less studied but have shown promise for smooth surfaces under highly controlled capture conditions. However, such methods suffer from inherent ambiguities and the depolarising effects of surface roughness. The method presented in this paper goes some way to overcome these limitations by fusing the most reliable information from PS and polarisation. PS is used with only two sources to deduce a constrained mapping of the surface normal at each point onto a 2D plane. Phase information from polarisation is used to deduce a mapping onto a different plane. The paper then shows how the full surface normal can be obtained from the two mappings. The method is tested on a range of real-world images to demonstrate the advantages over standalone applications of PS or polarisation methods. 
Dear reviewers and editor,

I am very grateful for the time and consideration that you have devoted to reviewing my paper. I was delighted to hear that the comments were mostly positive but also greatly appreciate the constructive feedback. I have addressed the issues raised and feel this has improved the paper significantly.

Please find my response to specific comments attached separately. I shall look forward to hearing your decision.

Best regards,

Gary Atkinson 


\section{*1. Response to Reviews}

Reviewer \#1:

"1. Do you evaluate the influence of individual steps in the proposed framework, e.g., the azimuth estimation? Which module is the major part regarding the overall accuracy?"

This is a very interesting question! I have conducted some experiments where either the polarisation data (phase and degree of polarisation) or photometric stereo data (intensity) were simulated. This showed clearly that, for simple surfaces at least, it is photometric stereo that is the main limitation. This additional analysis is now described near the end of Section 4.1 in lines 425 to 435 .

"2. How is the accuracy compared to utilizing conventional polarization methods such as [11][12]?"

I have compared some of the methods in the literature review more directly to the new method now (scattered throughout lines $61-78$ ). As is often the case, direct and perfectly fair comparisons are difficult due to unspecified details in certain papers and different hardware requirements (e.g. multi-view vs. multi-source). The most similar method however, is now compared numerically on line 74 . Lessquantitative pros and cons compared to the state-of-the-art are provided throughout the literature review.

"3. The hyperlinks of the figures \& references did not work in current pdf?"

Apologies for this. I believe it is a result of the LaTeX compiler in the submission system, which I do not have access to. The links are working when pdfLatex is used offline. I will make absolutely certain the links are all working in the published version.

Reviewer \#2:

"1. More evaluating in terms of normal accuracy

As the author also correctly claimed, that normal estimation accuracy cannot be completely reflected from the integrated surface. So, it makes more sense to show the normal comparison for Fig. 6 and Fig. 9. The author uses 12 error for several evaluations, but I think the angular error statistics makes the result more intuitively understandable in Figs. 4, 7 and $8 . "$

I agree that a little more consideration about the error metric used for each figure was necessary and have made some amendments accordingly:

- Fig 6 (now called Fig 7): I have left this particular figure as a depth graph because surface normal error (which both the reviewer and I agree is generally more representative) is evaluated in great detail afterwards. I feel it is useful to see overall depth errors at some point early in the results section. However, I can change this to normal angular error if the reviewer insists but I think that would not add much to the paper.

- Fig. 7 and 8 (now called 8 and 9): Converted to angular error as requested - good suggestion.

Fig. 9 (now 10): Converted to normal error as requested. I have also formatted this graph in the same way as Fig. 10 (now 12) for ease of comparison. However, I have also included the depth error graph as a new figure (Fig 11 in the new version) to demonstrate the effects of large angular errors on depth estimation.

- I'm a little confused about the reviewer's reference to Fig. 4 (now 5) as this is not a graph at all. However, I am confident that the changes above satisfy this comment.

"2. Specular reflection vs. specular interreflection

When analyzing Figs. 2 and 3, the author said in line 159 that the pi/2 flip is due to specular reflection. Specular reflection can indeed cause such a flip, but the scenario in Fig. 3 is mainly due to specular interreflection. Line 251 says both direct and inter-reflective specular reflection can cause such an effect. I suggest checking the consistency of this term, and make it clear throughout the paper. If possible, the author may also show an example due to specular reflection (e.g., by painting half of the diffuse sphere with some specular paint)." 
I agree with the reviewer that there is some inconsistency in terminology between "specularity", "interreflection", etc. I have therefore tidied up the terminology throughout the paper and used the terms "direct specularity" or "specular inter-reflection" throughout or simply used the term

"specular/specularity" where the text refers to either type. In addition, I have added a figure (Fig. 4 in the new version) to demonstrate a large direct specular reflection. This is explained in the last paragraph of Section 2.

\section{"3. Minor issues}

- Missing references:

[T. Ngo et al., Shape and Light Directions from Shading and Polarization, CVPR 15]: this paper combines photometric stereo and shape from polarization.

[W. Smith et al., Linear Depth Estimation from an Uncalibrated, Monocular Polarisation Image, ECCV $16]$ : this is one of the latest paper proposing a purely polarization based approach for complete normal estimation."

Thank you for suggesting these references. I strongly agree that these deserve a mention and have covered both in Section 1.1 (between lines 72 and 85).

"- It should be highlighted in the conclusion that a major limitation of using two-source photometric stereo is that it cannot deal with non-Lambertian materials (it could be quite sensitive to material reflectance variation due to too few light sources). But shape from polarization is not that sensitive to reflectance properties and thus can deal with much more broader range of material types. I think combining with two-source photometric stereo somehow sacrifices such an advantage of shape from polarization."

I agree that there is a limitation in this regard and that the first version of the paper is not as explicit about this as it should be. Consequently, I have added a brief discussion in the middle of the three paragraphs of the conclusion. It is worth noting however, that the claim that a polarisation-only approach "can deal with much more broader range of material types" than the proposed method is debatable: for example, roughness is one of the key physical features that can affect reflectance properties, but this would have a larger effect on polarisation than photometric stereo. In any case, I believe that the new text in the conclusion offers a fair evaluation of the situation. 
A method for surface normal recovery using photometric stereo and polarisation

Two source photometric stereo used for 2D normal estimation

Polarisation information used for azimuthal angle estimation

Unknowns in normals due to ambiguities are resolved via region growing across surface

Method is proven robust to specular inter-reflections and evaluated extensively 


\title{
Polarisation Photometric Stereo
}

\author{
Gary A. Atkinson \\ Bristol Robotics Labratory \\ Department of Engineering Design and Mathematics \\ University of the West of England, Bristol \\ $B S 161 Q Y$ UK
}

\begin{abstract}
This paper concerns a novel approach to fuse two-source photometric stereo (PS) data with polarisation information for complete surface normal recovery for smooth or slightly rough surfaces. PS is a well-established method but is limited in application by its need for three or more well-spaced and known illumination sources and Lambertian reflectance. Polarisation methods are less studied but have shown promise for smooth surfaces under highly controlled capture conditions. However, such methods suffer from inherent ambiguities and the depolarising effects of surface roughness. The method presented in this paper goes some way to overcome these limitations by fusing the most reliable information from PS and polarisation. PS is used with only two sources to deduce a constrained mapping of the surface normal at each point onto a $2 \mathrm{D}$ plane. Phase information from polarisation is used to deduce a mapping onto a different plane. The paper then shows how the full surface normal can be obtained from the two mappings. The method is tested on a range of real-world images to demonstrate the advantages over standalone applications of PS or polarisation methods.
\end{abstract}

Keywords: Polarisation, photometric stereo, 3D reconstruction

\section{Introduction}

Three-dimensional surface reconstruction/analysis has been a major goal of computer vision for many years. There have a plethora of methods proposed 
in the last few decades, each with their unique pros and cons. For example,

5 binocular stereo [1] applies at any range and is inexpensive but inappropriate for featureless surfaces. By contrast, time-of-flight cameras 2 are known to operate on a variety of scenes at high speed but suffer at long range. This paper uses a fusion of photometric stereo (PS) and polarisation vision aimed at featureless surfaces at close range: a goal that typically requires expensive methods that use lasers or active illumination, else only operate at low resolution.

The proposed method initially applies a form of two-source PS to estimate part of the surface normal. This is much more applicable to real world applications than most PS methods that require three or more sources on different illumination planes. Polarisation information is then used in conjunction with the PS-based estimates in order to fully constrain the normals at each pixel. The paper therefore represents a significant step forward towards commercial robotics applications able to exploit a largely untapped area of light: that of its polarisation state.

This paper starts by reviewing the state-of-the-art in three-dimensional surface estimation using polarisation. Then, in Section 2, the key background theory from Fresnel Theory and polarisation imaging is provided. The novel reconstruction algorithm is described in detail in Section 3. Experimental results and conclusions are provided in Sections 4 and 5 respectively.

\subsection{Related work}

One of the early proposed methods for surface shape estimation was that of shape-from-shading, which aims to acquire geometry from an image using intensity information only [3]. Due to its single-view and passive nature, it is an attractive goal and does not suffer from the limitations of the above examples (binocular stereo and time of flight). Unfortunately, the method is known to be ill-posed due to the bas-relief ambiguity and other reasons 4. PS was proposed as a means to overcome the ambiguities at the expense of the need for three or more light sources, of known direction, illuminated separately with an image captured for each. Only relatively recently however has this 
method become useful in many applications due to the relative ease of high speed camera-illumination synchronisation [5]. However, the applications are still highly limited due to the need for at least three sources. For example, for many land-based robotics applications, it is easy to include two light sources (to the far-left and far-right of the robot), but it is difficult to position a source high above the ground. That said, Zhang et al. [6] apply geometric assumptions 40 to force a solution for smooth surfaces with two-source PS.

A less-studied approach to $3 \mathrm{D}$ vision problems is that of polarisation vision. That is, the use of the polarisation state of reflected light to deduce surface information [7]. As with the field of hyperspectral imaging, the method taps into an entire set of information about the incoming light (polarisation state/spectral 45 composition) that a standard greyscale or RGB camera is unable to. Thus, one may argue that a great deal of potentially useful information about the incoming light is lost in standard vision techniques. Of course, the additional information available from polarisation and hyperspectral imaging systems comes at the cost of more expensive and often slower capture hardware.

Most previous computer vision research using polarisation uses Fresnel theory applied to specular reflection [8]. The theory tells us that initially unpolarised illumination undergoes a partial linear polarisation process upon reflection from surfaces. The specific properties of the polarisation correlate to the relationship between the surface orientation and the viewing direction [7, 9].

${ }_{55}$ Unfortunately, there are inherent ambiguities present (see Section 2) and the refractive index of the target is typically required. Further, different equations are required to model reflection if a diffuse component is present.

Miyazaki et al. [10, Atkinson and Hancock [11] and Berger et al. [12] all use multiple viewpoints to overcome the ambiguity issue. In [10, specular reflection is used on transparent objects - a class of material that causes difficulty for most computer vision methods. In [11, a patch matching approach is used for diffuse surfaces to find stereo correspondence and, hence, 3D data. Results are of comparable quality to this paper, but cannot easily handle specularities/interreflections. In [12, an energy functional for a regularization-based stereo vision 
65 is applied. Taamazyan et al. [13] use a combination of multiple views and a physics-based reflection model to simultaneously separate specular and diffuse reflection and estimate shape (Miyazaki et al. 14 also separate reflection components but only as a pre-processing step).

Drbohlav and Šára [15, 16] use PS (as in this paper) but with linearly polarized incident illumination. Atkinson and Hancock [17] also use PS but with more light sources and less resilience to inter-reflections than the method of this paper. Ngo et al. [18] propose a similar combined polarisation/two-source PS method to the one presented in this paper with optimal results reported to be of the order $2^{\circ}$ error from ground truth (this paper manages of $3-4^{\circ}$ ). However, the Ngo paper focuses on estimating the lighting directions rather than addressing complications due to specularities/inter-reflections (which was a key motivation here).

Garcia et al. 19] use a circularly polarised light source to overcome the ambiguities while Morel et al. 20] extend the methods of polarisation to metallic surfaces by allowing for a complex index of refraction. Smith et al. 21] assume a known constant refractive index and albedo but are able to pose the problem as a system of linear equations. This allows to directly deduce depth up to a concave/convex ambiguity in the presence of both diffuse and specular reflection. Furthermore, the method is successfully applied to conditions of uncontrolled 85 illumination and only relies on a single view.

In an effort to overcome the need for the refractive index, Miyazaki et al. constrain the histogram of surface normals [14 while Rahmann and Canterakis [22, 23] use multiple views and the orientation of the plane of polarisation for correspondence. Huynh et al. 24] actually estimate the refractive index using spectral information. Kadambi et al. 25] propose a method to allow polarisation information to be combined with alternative depth capture methods resulting in overall improved reconstructions. 


\subsection{Contribution}

The motivation for this paper relates to the following weaknesses of existing

methods in related areas: firstly the need for three light sources in PS; secondly the multiple ambiguities in polarisation; and thirdly the complexities due to specular inter-reflections and diffuse reflection in both PS and polarisation. The contributions are as follows:

- Exploit the advantages of PS methods but using only two sources.

- Reduce the dependency of PS on light source knowledge.

- Use a combination of PS and polarisation data with a novel region growing algorithm to deduce the full 3D surface normals of an object in the presence of specular inter-reflections (assuming that the majority of the surface is not specularly reflecting). The method is also somewhat robust to direct specularities, depending on their relative intensity compared to the diffuse component.

- Avoid the need to know or estimate the refractive index.

As stated earlier, it is thought that overcoming these limitations allows a significant step towards the usage of polarisation information in real-world robotics applications.

\section{Polarisation Vision}

This paper is based on the premise that light undergoes a partial polarisation process upon reflection from smooth surfaces. Consider the experimental arrangement shown in Fig. 1, which is used for all of this work. Fresnel reflectance theory [8] is able to quantify the polarisation process that occurs when initially unpolarised incident light is reflected towards the camera. The reflected light can be parametrised by three values. First is the intensity, $I$, of the light. Second is the phase angle, $\phi$, which defines the principle angle of the electric field component of the light as shown in the figure. Finally, the degree of polarisation, $\rho$, indicates the level of polarisation from 0 (unpolarised) to 1 (completely linearly polarised) [26]. 


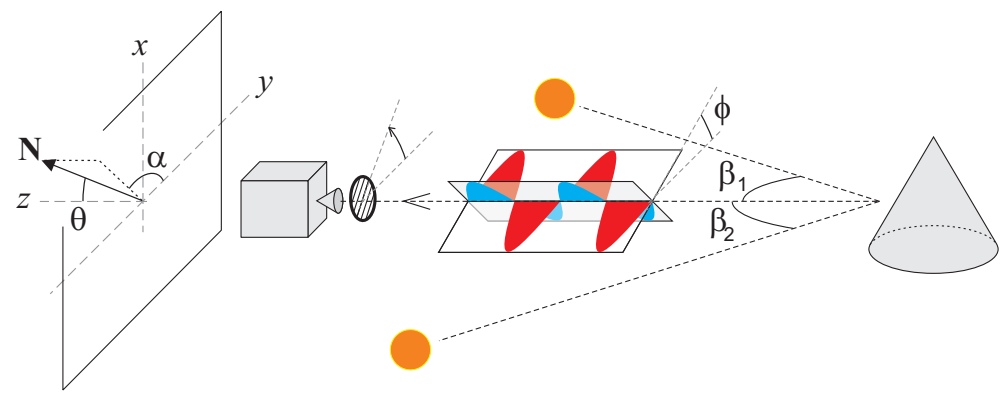

Figure 1: Arrangement and definitions. Two polarisation images are acquired using the camera and rotating polariser. Each image has a different source illuminated. The surface normal, $\mathbf{N}$, is defined by its zenith angle, $\theta$, and azimuth angle, $\alpha$.

As explained elsewhere in the literature [7, 26, for specularly reflected light the phase angle aligns perpendicularly to the projection of the surface normal onto the image plane. Assuming that the Wolff subsurface scattering model [27] applies, diffuse reflection causes parallel alignment. Since there is no distinction in phase angle shifts of $\pi$ radians, there are therefore four possible surface azimuthal angles (defined in Fig. 1) for an unknown reflectance type:

$$
\alpha \in\left[\phi, \phi+\frac{\pi}{2}, \phi+\pi, \phi+\frac{3 \pi}{4}\right] \quad \text { where } \quad 0 \leq \phi<\pi
$$

The degree of polarisation contains information principally related to the zenith angle of the surface. Unfortunately, the relationship between zenith angle and degree of polarisation varies substantially between specular and diffuse reflection and depends on the refractive index of the reflecting surface, which is typically unknown [26]. For this reason, its use for surface normal estimation is avoided in this paper. The method does however use the degree of polarisation as a measure of reliability of the phase data: any phase data with a corresponding degree of polarisation below a threshold (fixed at 1\%) is deemed unreliable due their correspondingly high associated noise levels.

The method described in the next section requires two polarisation images of an object for input (a single polarisation image comprises a separate intensity, phase and degree of polarisation value for each pixel). There are now several commercially available cameras that capture such data directly such as the 
Fraunhofer "POLKA" 28]. The POLKA has a sensor divided into $2 \times 2$ cells, with each element sensitive to light of a given electric field orientation $\left(0,45^{\circ}\right.$, $90^{\circ}$ and $135^{\circ}$ ). If the intensities measured for these angles are called $I_{0}, I_{45}$, $I_{90}$ and $I_{135}$ respectively, then the polarisation data for each cell is calculated via the Stoke's parameters $S_{0}, S_{1}$ and $S_{2}$ (assuming no circular polarisation is present) [8]:

Stoke's parameters:

$$
\begin{gathered}
S_{0}=\frac{I_{0}+I_{45}+I_{90}+I_{135}}{2} \\
S_{1}=I_{0}-I_{90} \\
S_{2}=I_{45}-I_{135}
\end{gathered}
$$

Polarisation image data:

$$
\begin{gathered}
I=S_{0} \\
\phi=\frac{1}{2} \arctan _{2}\left(\frac{S_{2}}{S_{1}}\right) \\
\rho=\frac{\sqrt{S_{1}^{2}+S_{2}^{2}}}{S_{0}}
\end{gathered}
$$

where $\arctan _{2}$ is the four quadrant inverse tangent [29].

Raw data for this paper were captured using a Dalsa Genie HM1400 camera with a motor-controlled circularly polarising filter in front of the lens. The motor rotated the filter to angles of $0,45^{\circ}, 90^{\circ}$ and $135^{\circ}$ between each image capture, after which (27) were applied to calculate the polarisation image; following a similar method to the POLKA. A circular filter was used, rather than linear, in order to minimise effects of any inter-reflections in the lens (Schneider KMP-IR Xenoplan 23/1,4-M30,5) [30. The rotating polariser method is clearly much slower (but cheaper) than using the POLKA (or similar products) and thus restricts application to stationary objects. However, the rest of the paper is independent of the capture method and so could easily be applied to moving objects if such cameras were used with this method in the future.

Examples of the three components of the polarisation image for a white snooker ball are shown in Figs. 2 to 4 . Note that the intensity is normalised to the range $[0,1]$. For Fig. 2, the capture conditions were ideal, with the 
ball illuminated by a single small white LED placed close to the camera and blackout curtains surrounding the ball to diminish all inter-reflections from the environment. The reflection is therefore of diffuse type throughout the surface and so the first or third solutions to (1) must be true for all pixels.

For Fig. 3, a plain matte white board was placed behind the ball. A sudden phase shift is present near the occluding contours of the ball due to an interreflection from the board. Since this region is effectively undergoing a specular inter-reflection, the second or fourth solutions to (1) are true. By contrast, the first or third solutions are true for the rest of the ball, which is exhibiting diffuse reflection. Note also, that the degree of polarisation is higher near the inter-reflection on the right-hand side of the ball. This is also predicted by the reflection theory but is of less significance to this paper [26]. The noise-ridden background to Fig. 3 is due to the very low degree of polarisation for the board and the shadow cast by the ball; neither of which are of significance here.

The polarisation image in Fig. 4 includes an extended light source located to the upper-right of the ball. The phase shift is clearly present here, demonstrating the specular nature of the direct reflection. Careful examination of the degree of polarisation demonstrates some inter-reflections from the laboratory are present just below the main specularity. Since the intensity from these inter-reflections is very low, the dominant reflection type is diffuse there.

\section{Method}

The approach can be broadly divided into the following steps assuming that the starting point is two full polarisation images corresponding to the two light source locations shown in Fig. 1.

1. Extract estimates of 2D $(y-z)$ surface normals from two-source PS.

2. (a) Extract estimates of $2 \mathrm{D}(x-y)$ surface normals from polarisation aided by PS.

(b) Disambiguate spurious data or specular inter-reflections (or, potentially, direct specularities) using region growing. 
(a)

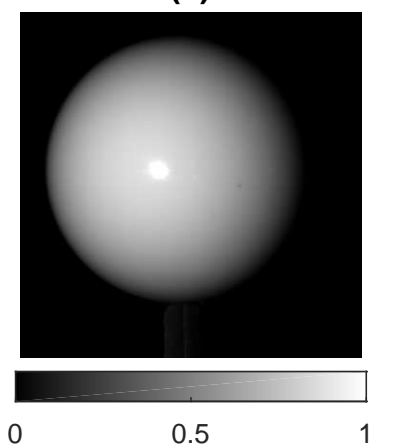

(b)

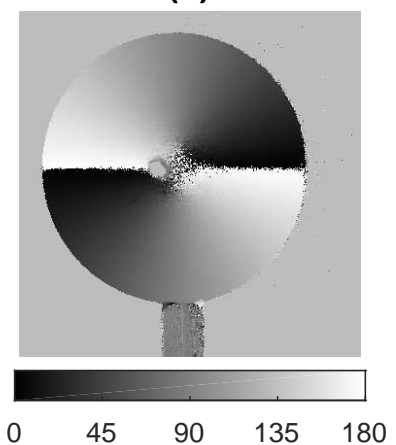

(c)

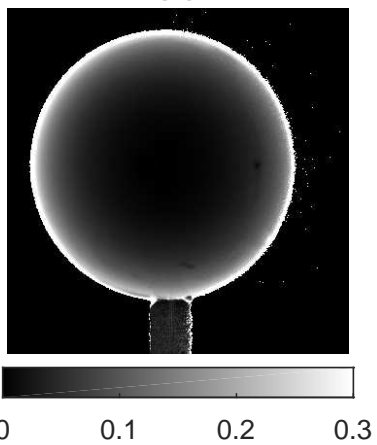

Figure 2: Example polarisation image of a white snooker ball with no inter-reflections and only one specularity (which is saturated in the image). (a) Intensity $I$, (b) phase angle $\phi$, (c) Degree of polarisation, $\rho$.

(a)

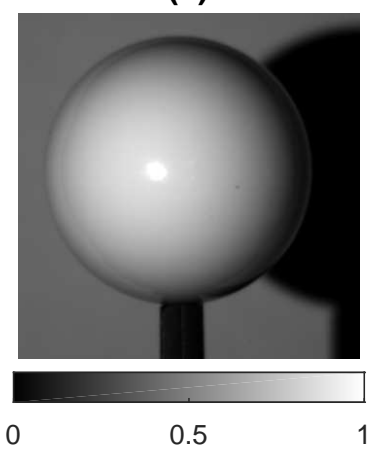

(b)

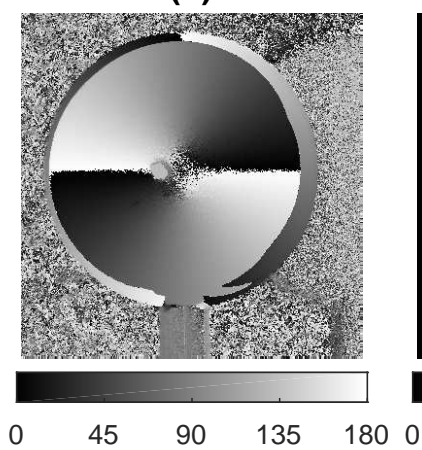

(c)

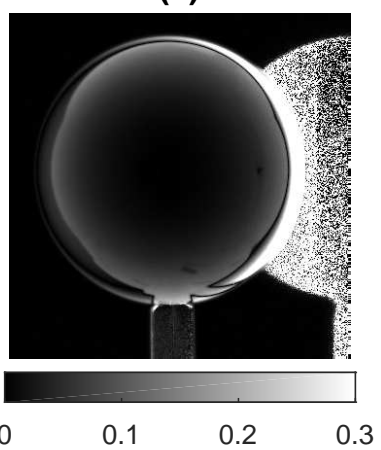

Figure 3: Example polarisation image of a white snooker ball with specular inter-reflections from a white board behind the ball. Otherwise, conditions were the same as in Fig. 2 (a) Intensity $I$, (b) phase angle $\phi$, (c) Degree of polarisation $\rho$. The degree of polarisation values above 0.3 (right side of ball) are all shown white here to improve the clarity of the rest of the figure. 
(a)

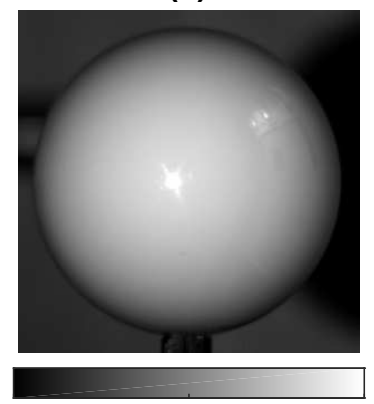

0 (b)

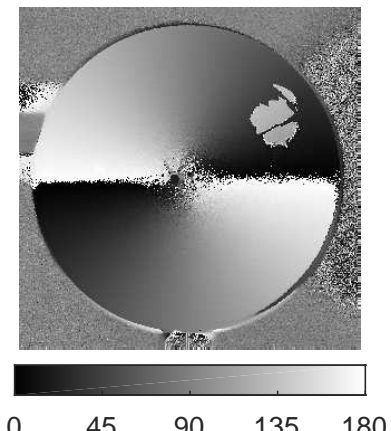

(c)

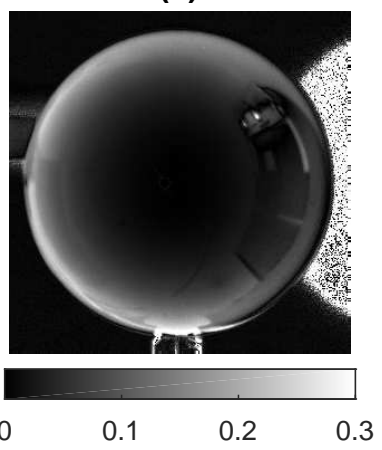

Figure 4: Example polarisation image of a white snooker ball with a direct specularity from an extended source and a sequence of uncontrolled environmental inter-reflections on the right-hand side. (a) Intensity $I$, (b) phase angle $\phi,(\mathrm{c})$ Degree of polarisation $\rho$.

3. Combine data from each modality to obtain 3D $(x-y-z)$ surface normals.

The first step essentially applies a 2D version of PS to obtain a 2D surface normal at each pixel. This is not merely a projection of the $3 \mathrm{D}$ normal onto the $y-z$ plane but rather a vector representing one particular degree of freedom of the orientation of the surface at each point. The second step uses the polarisation phase angle of the incoming light and assumes diffuse reflection (for now) to estimate a $2 \mathrm{D}$ version of the surface normal in the $x-y$ plane. PS data is applied to disambiguate most of these vectors but region growing is needed where specularities occur or where previous disambiguation is deemed incorrect (as defined in Section 3.2.2). Finally, the information above is combined to form a $3 \mathrm{D}$ surface normal map.

\subsection{Application of PS}

The aim here is to use the method of PS to estimate a 2D normal in the $y-z$ plane at each point. To do this, the original methodology of Woodham [31] is adapted to two images in order to obtain a set of $n$ normals as follows:

$$
\left\{\mathbf{N}_{i}^{(\mathrm{ps})}=\left[\begin{array}{c}
N_{y, i}^{(\mathrm{ps})} \\
N_{z, i}^{(\mathrm{ps})}
\end{array}\right]\right\}_{i=1}^{n} \leftarrow\left[\begin{array}{cc}
-\cos \left(\beta_{R}\right) & \cos \left(\beta_{L}\right) \\
\sin \left(\beta_{R}\right) & -\sin \left(\beta_{L}\right)
\end{array}\right]\left[\begin{array}{c}
I_{L, i} \\
I_{R, i}
\end{array}\right] \forall i
$$


where the angles $\beta_{L}$ and $\beta_{R}$ are defined in Fig. 1 and the suffix "ps" is a reminder that these estimates are from photometric stereo. The " $\leftarrow$ " symbol is used throughout this paper to refer to variable assignment. The vectors are then normalised into unit vectors in preparation for fusion with the polarisation data.

One of the aims of this paper is to ensure that the method is robust to disruptions in illumination. For example, standard PS yields poor results if one source appears much brighter than the other or if the distance to the target object varies (as this affects the light source vector). The results for this paper were obtained with accurately positioned illumination so this potential issue does not manifest. However, it is possible to use the phase information from polarisation to calibrate the light source intensities assuming a linear (or at least known) camera response profile. This is done by scaling the intensities 225 such that areas where $\phi=90^{\circ}$ or $270^{\circ}$ have equal $I$ values. Further, it is worth noting at this point that the distance-to-target issue is less pronounced here than standard PS since only the $z$-component of the normal is affected by incorrect source vectors.

\subsection{Application of polarisation vision}

This section describes the method to estimate $2 \mathrm{D}$ normals in the $x-y$ plane. It assumes that the method for polarisation image acquisition described in Section 2 has been applied to arrive at corresponding intensity, phase and degree of polarisation values for all $n$ pixels: $\left\{I_{i}, \phi_{i}, \rho_{i}\right\}_{i=1}^{n}$. This section also makes use of $\left\{\mathbf{N}_{i}^{(\mathrm{ps})}\right\}_{i=1}^{n}$. This part of the algorithm is in two parts, the code for which is 235 summarised in Algorithm 1.

The raw data yields two estimates of polarisation values for each pixel (one corresponding to each light source direction). In theory, the data should be identical between each polarisation image, except for the locations of specularities and shadows. The proposed algorithm first forms a new polarisation image corresponding to what is deemed the most reliable data for each pixel. Two methods were considered for this: (1) use the polarisation data from the image 
corresponding to the highest intensity at each point, (2) use the polarisation data from the image corresponding to smallest deviation between raw data and the transmitted radiance sinusoid [26]. While the latter approach is perhaps more technically sound, it was experimentally determined that the final quality of results were very similar between the two approaches. For this reason, the former more computationally efficient approach was used for the remainder of the research.

\subsubsection{Initial azimuth estimation}

This is covered by the first three lines of Algorithm 1. The first line applies a sharpening operator to the phase data. The reason for this that the subsequent specularity detection/disambiguation algorithm ("localAlign") is more reliable in the presence of sharp transitions between specularities and diffuse regions. An alternative to this is to increase the definition of a neighbourhood in the subsequent function, allowing the method to bridge over any gradual transitions.

The method makes the initial assumption that all surface normal projections

\begin{tabular}{l} 
Algorithm 1 Input: $\left\{\phi_{i}, \rho_{i}, \mathbf{N}_{i}^{(\mathrm{ps})}\right\}_{i=1}^{n}$ Output: $\left\{\mathbf{N}_{i}^{(\mathrm{po})}\right\}_{i=1}^{n}$ \\
\hline 1: $\left\{\phi_{i}\right\} \leftarrow \operatorname{sharpen}\left(\left\{\phi_{i}\right\}\right)$ \\
2: $\left\{\alpha_{i}\right\} \leftarrow\left\{\begin{array}{cc}\phi_{i} & \forall i \mid\left(N_{y, i}^{(\mathrm{ps})}<0 \wedge \phi_{i}>\frac{\pi}{2}\right) \vee\left(N_{y, i}^{(\mathrm{ps})}>0 \wedge \phi_{i}<\frac{\pi}{2}\right) \\
\phi_{i}+\pi & \text { otherwise }\end{array}\right.$ \\
3: $\left\{\mathbf{N}_{i}^{(\mathrm{po})}=\left[\begin{array}{c}N_{x, i}^{(\mathrm{po})} \\
N_{y, i}^{(\mathrm{po})}\end{array}\right]\right\}_{i=1}^{n} \leftarrow\left[\begin{array}{c}-\sin \alpha_{i} \\
\cos \alpha_{i}\end{array}\right] \forall i$ \\
4: $\left\{R_{i}\right\}_{i=1}^{n} \leftarrow\left\{\begin{array}{cc}1 & \forall i \mid \text { isBorderPixel }(i) \vee \rho_{i}<0.01 \\
0 & \text { otherwise }\end{array}\right.$ \\
5: while $[$ More seed points?] do \\
6: $\quad j \leftarrow$ Get seed point \\
7: $\quad\left\{\mathbf{N}^{\text {(po) }\} \leftarrow \text { localAlign }\left(\left\{\mathbf{N}^{(\mathrm{po})}, R\right\}, j\right)}\right.$ \\
\hline
\end{tabular}


onto the $x-y$ (image) plane are aligned parallel to, or anti-parallel to, the phase angle, as predicted by the theory for diffuse reflection covered in Section 2 Line 2 sets the azimuthal angle, $\alpha$, of each point accordingly. Whether said projections are parallel or anti-parallel depend on the best match to the estimated 2D normal from PS. Line 3 simply generates a set of $2 \mathrm{D}$ surface normals on the $x$-y plane using the calculated azimuth angles. The suffix "po" reminds us that these estimates are primarily from polarisation.

\subsubsection{Final azimuth estimation}

This is covered by the last four lines of Algorithm 1. It is expected that most of the normals will be correct at this point. However, it is likely that some regions of the image will be incorrect due to one of the following reasons:

- Diffusely reflecting regions with incorrect disambiguation. This is typically where $N_{y}^{(\mathrm{ps})} \approx 0$, meaning the initial disambiguation is not robust. These regions have an azimuth error or $\pi$ radians.

- Specular (direct or inter-reflective) regions. In these areas the azimuth angle error is $\pi / 2$ radians, as predicted by the theory described in Section 2.

Lines 4 to 7 of Algorithm 1 are intended to address these possibilities, while enforcing (1). First, a set of pixels, $\left\{R_{i}\right\}_{i=1}^{n}$ are determined that should not be further considered by the algorithm. In the first instance, these pixels correspond to:

- Image border pixels: purely to avoid slowing down the algorithm with numerous conditional statements.

- Those with very low $(<1 \%)$ degree of polarisation: since these areas are deemed to have too much noise to be of use.

Next, a seed point is chosen. This can easily be done either manually or randomly using a point of high confidence (i.e where the degree of polarisation is high and either $N_{y}^{(\mathrm{ps})} \ll 0$ or $\left.N_{y}^{(\mathrm{ps})} \gg 0\right)$. One weakness here however, is that 


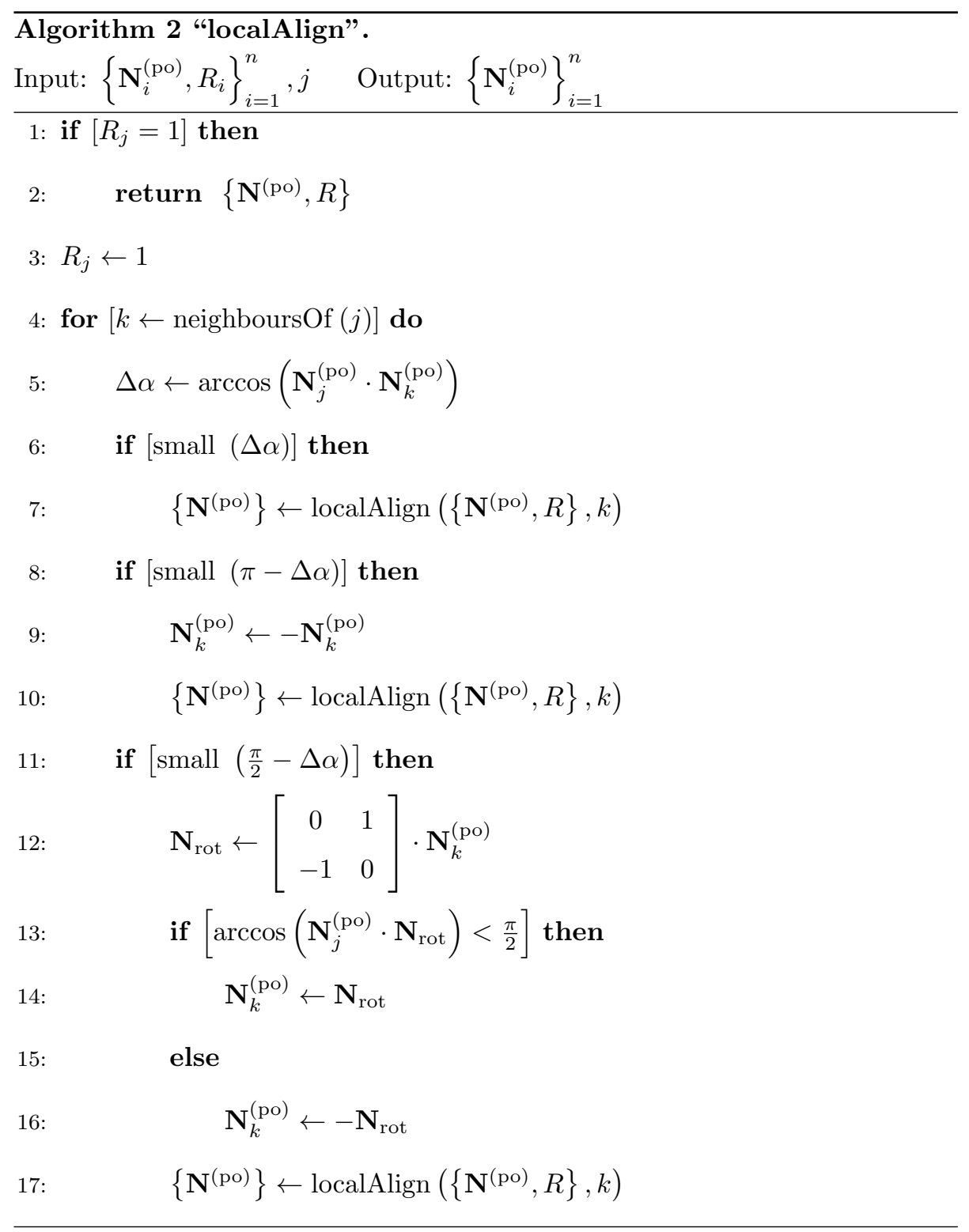

this point must be of diffuse reflection so may benefit from some heuristics-based selection in future work. Assume for now that only one seed point is needed, but note that the code permits more if necessary (e.g. for complicated shapes). The rest of the process involves a recursive call to a region growing function, which progressively aligns spurious normals according to the constraints of (1). 

varied between $5^{\circ}$ and $10^{\circ}$.

\subsection{Fusion of data into full 3D surface normals}

The methods from the previous two sections give two sets of $2 \mathrm{D}$ surface normals: $\left\{\mathbf{N}^{(\mathrm{ps})}\right\}$ on the $y-z$ plane and $\left\{\mathbf{N}^{(\mathrm{po})}\right\}$ on the $x-y$ plane. These can 315 normalised such that the following is true for each point:

$$
N_{x}^{(\mathrm{po})^{2}}+N_{y}^{(\mathrm{po})^{2}}=1=N_{y}^{(\mathrm{ps})^{2}}+N_{z}^{(\mathrm{ps})^{2}}
$$


where the subscripts $i$ are omitted for the sake of brevity.

Denote the $3 \mathrm{D}$ surface normal at a particular point $\mathbf{N}=\left[N_{x}, N_{y}, N_{z}\right]^{T}$. Further, choose the components estimated by polarisation for $x$ and $y$ so there is only one unknown, $N_{z}$ :

$$
\mathbf{N}=\left[N_{x}, N_{y}, N_{z}\right]^{T}=\left[N_{x}^{(\mathrm{po})}, N_{y}^{(\mathrm{po})}, N_{z}\right]^{T}
$$

Normalising this vector to unit length gives:

$$
\mathbf{n}=\frac{\left[N_{x}^{(\mathrm{po})}, N_{y}^{(\mathrm{po})}, N_{z}\right]^{T}}{\sqrt{1+N_{z}^{2}}}=\left[n_{x}^{(\mathrm{po})}, n_{y}^{(\mathrm{po})}, n_{z}\right]^{T}
$$

This can then be re-normalised such that $n_{y}^{(\mathrm{po})^{2}}+n_{z}^{2}=1$, matching the form of the $2 \mathrm{D}$ estimate from PS as in (9). For the $z$ component specifically:

$$
\frac{n_{z}}{\sqrt{n_{y}^{(\mathrm{po})^{2}}+n_{z}^{2}}}=N_{z}^{(\mathrm{ps})}
$$

Substituting the components of $\mathbf{n}$ from 11 and simplifying:

$$
N_{z}^{(\mathrm{ps})}=\frac{N_{z}}{\sqrt{N_{y}^{(\mathrm{po})^{2}}+N_{z}^{2}}}
$$

325 Rearranging:

$$
N_{z}=\left|N_{y}^{(\mathrm{po})}\right|\left(\frac{1}{N_{z}^{(\mathrm{ps})^{2}}-1}\right)^{-1 / 2}
$$

where the $|\cdot|$ sign is used since $N_{z}$ must be positive. This means that the only unknown in 10 is resolved and so the surface normal is fully determined.

As stated earlier, the regions of the images where the degree of polarisation is less that $1 \%$ are not used in the algorithm to ensure robustness. For the results in the next section, bi-cubic interpolation was used to estimate the normals for these areas. It was also found that improvements could be made by interpolating over regions of very low $N_{y}$, although this was kept to a minimum. It is acknowledged however, that more sophisticated methods from the shape-from-shading literature 3 may be more appropriate for future work. 
The normals can be converted to depth using any of a number of integration methods 32]. Since it is not the aim of this paper to develop a new integrator, the well-established Frankot-Chellappa method [33 is primarily used for this step. The method is fast and highly robust to noise, but can suffer from over-smoothing. An alternative method that does not over-smooth is briefly considered in Section 4.3

\section{Results}

This section contains a detailed analysis of the performance of the method for a range of target objects. In the first instance, a thorough numerical evaluation for the surface normals and height estimations are carried out for a white snooker ball under varying conditions and compared to ground truth and baseline methods. Subsequently, a range of more challenging shapes and textures are considered.

\subsection{Spherical objects}

Consider Fig. 2, the polarisation image for a white snooker ball captured in ideal conditions as described in Section 2. The angle between the camera and light sources was $\beta_{L}=\beta_{R}=19.6 \pm 0.5^{\circ}$. One hundred images were captured at each of the four polariser angles $\left(0,45^{\circ}, 90^{\circ}\right.$ and $135^{\circ}$, aligned with resolution to the nearest degree) and the mean intensity used at each pixel to minimise noise. As expected, the phase angle directly relates to the surface azimuth up to a $180^{\circ}$ ambiguity and the degree of polarisation is highest near the occluding contours [26].

As mentioned earlier in this paper, and elsewhere in the literature, among the issues facing polarisation-based computer vision algorithms are the complications caused by the simultaneous presence of specular and diffuse reflection. To test the robustness of the method proposed here, a second polarisation image was captured in exactly the same conditions as for Fig. 2 but with a large planar matte white surface placed at approximately $128 \mathrm{~mm}$ behind the target 

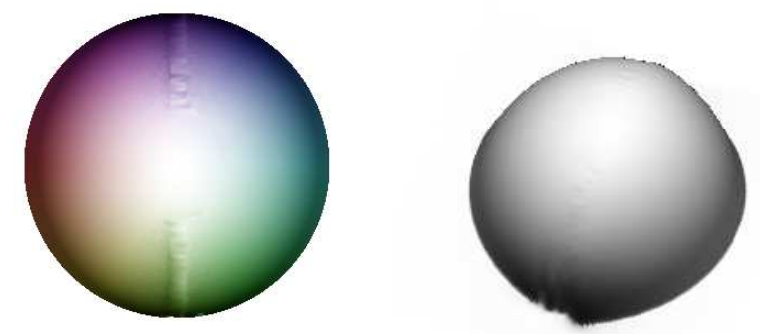

Figure 5: Surface normals and depth estimated from the polarisation image shown in Fig. 2 (and its right-illuminated counterpart). Surface normals are encoded by colour (azimuth) and saturation (zenith).

object. The resulting image is shown in Fig. 3 , as also discussed in Section 2 The specular inter-reflection can only just be seen in the intensity image, yet the $90^{\circ}$ phase shift and increased degree of polarisation make it clear in those two components of the polarisation image.

The results of applying the surface normal estimation algorithm to the polarisation images shown in Figs. 2 and 3 are shown in Figs. 5 and 6 , respectively. The results are qualitatively good although the region of interpolation (described in Section 3.3) near the top-centre and bottom-centre of the normal maps is clearly apparent. Note that the data has been cropped here so only the object itself is being integrated.

Fig. 7 shows a comparison of the profile of the reconstructions from Figs. 5 and 6 to ground truth data. To aid comparison, the profiles are aligned such that the tops of each curve are touching. Very similar results were found for pink and yellow balls, while blue and green results were slightly poorer due to their lower brightness causing higher noise levels.

In order to optimise the surface normal estimates with regards to the light source separation, both simulated and real-world data were analysed for varying light source angles. For the simulated data, images were generated assuming no inter-reflections/specularities were present and a range of illumination angles, $\beta$, between $5^{\circ}$ and $25^{\circ}$. Gaussian noise was also added with standard deviations 

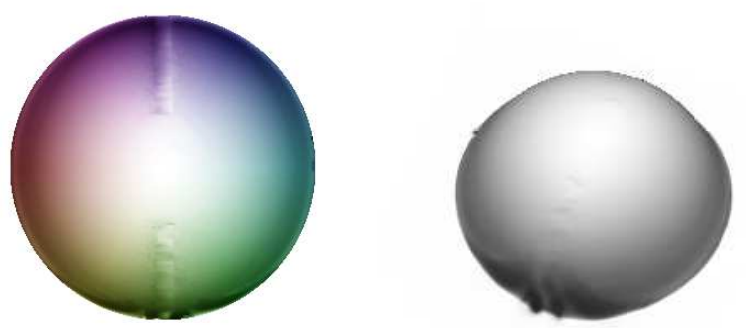

Figure 6: Surface normals and depth estimated from the polarisation image shown in Fig. 3 (and its right-illuminated counterpart). Surface normals are encoded by colour (azimuth) and saturation (zenith).

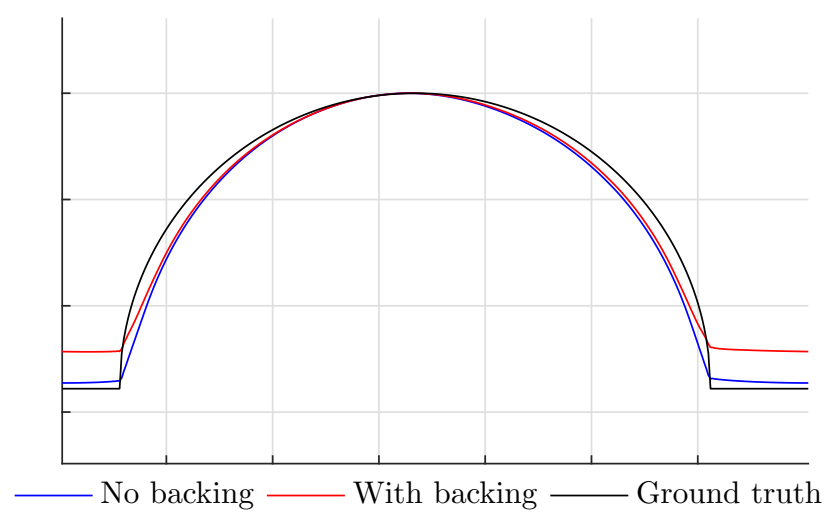

Figure 7: Comparison between the estimated depth profile of the snooker ball and ground truth for the data represented in Figs. 5 and 6 


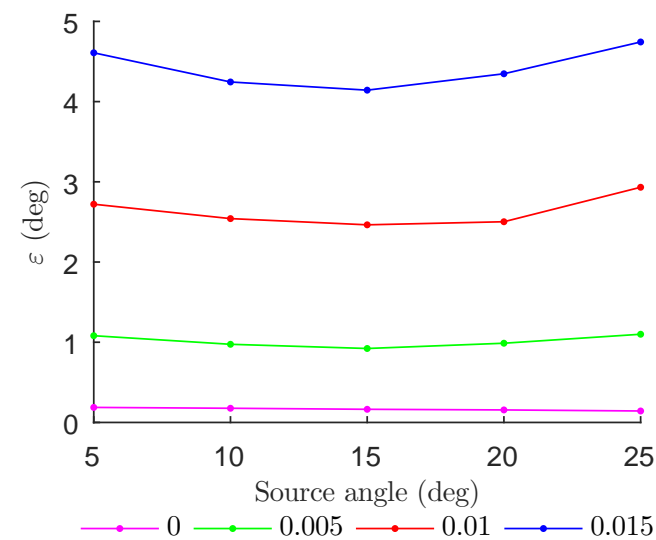

Figure 8: Surface normal errors using synthetic data with varying levels of Gaussian noise added (standard deviations shown in legend).

ranging from 0 (no noise) to 0.015 (typical for the camera settings used). The results are shown in Fig. 8, where the performance metric used is the median angular error, $\varepsilon$, between the method and ground truth. This metric has been chosen since the more obvious choice of height residuals would also be assessing the integration method used, which is not the contribution of the current paper.

The results to the analysis of simulated data show the following:

- The surface normals are not perfectly estimated even with no noise present as a result of small surface regions being interpolated over. There may also be some edge-effects degrading results slightly.

- There is relatively little variation in performance with illumination angle for most noise levels.

- There is an optimum illumination angle of about $15^{\circ}$ for higher noise levels. This is due to the fact that (1) at very small angles the difference between intensity images is very small which is poor conditioning for PS, and (2) at large angles major areas of shadow are present.

Experimental results for varying the illumination angle are shown in Fig. 9 Errors in both surface angle, $\varepsilon$, and root-mean-square height are shown although 
(a)

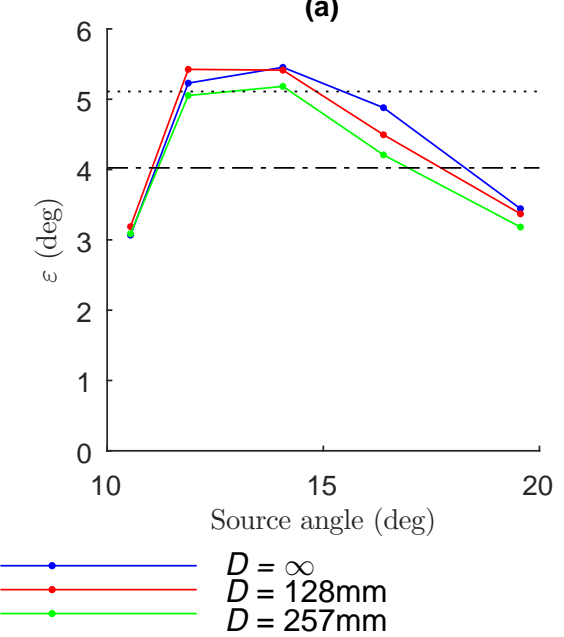

(b)

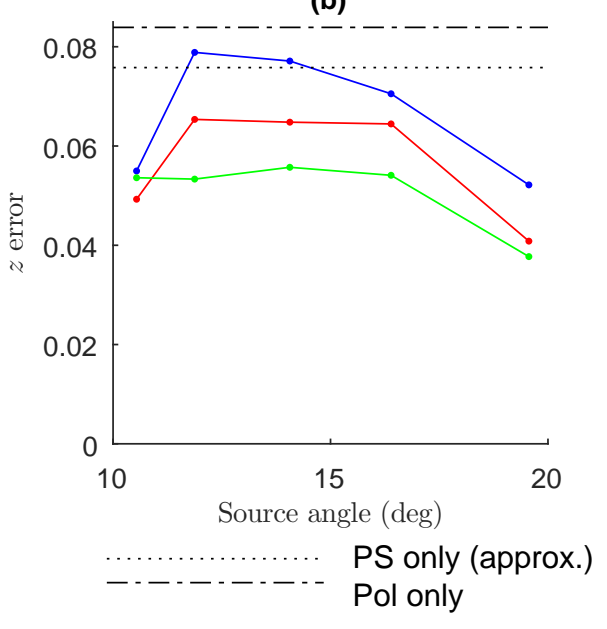

Figure 9: (a) Angular errors and (b) height errors for the white snooker ball with a white board at various distances behind the ball, $D$. Height values are in units such that a value of 1 corresponds to a distance of one radius. Best case results for PS-only and polarisation-only are also indicated.

400 ingly, the magnitudes of the errors are slightly higher than for simulated data for most cases. Surprisingly however, the trend towards an optimum separation is reversed for the real data. It is not entirely clear why this should be the case but repeated experiments have yielded similar trends. It was therefore decided to use $20^{\circ}$ illumination angles for the remainder of the research (higher values than this were neither practical for the experimental rig being used nor for most real-world applications).

Fig. 9 also shows results with the white board re-introduced at several distances and the baseline results for standard photometric stereo and single-source polarisation. The results with the board present show a strong performance by the method at overcoming specular inter-reflections, which is one of the key strengths of the method. Indeed, there is evidence that in some cases the reconstructions may be even better when strong specular behaviour is present. For standard photometric stereo, four sources were used at the illumination angle 

and $z$-components only for compactness) using 1, 5, 20 and 100 images. At first sight, the quality of results is poor when only few images are used. However, the increased noise has a relatively low impact on surface height reconstruction, as shown in the Fig. 11. This is due to the facts that (1) the Frankot-Chellappa

that gave the best surface normal estimates for that method (which was experimentally determined to be $25^{\circ}$ ). For the single-source polarisation baseline, a single polarisation image was used with the illumination source as close to the camera as possible $\left(\beta_{L}=\beta_{R} \approx 5^{\circ}\right.$ in practice) in order to minimise shadowed regions. Regions of the image were manually selected in order to disambiguate the azimuth angles and the zenith angles were calculated according to the Wolff diffuse reflection model with a refractive index of 1.5 [7. At first sight, the baseline polarisation method appears comparable to the novel method, but it is reiterated that the novel method is superior as it does not require manual disambiguation nor knowledge of refractive index.

For the case with no board present behind the ball, a test on partially simulated data was conducted in order to analyse the relative effect of the PS and polarisation elements of the method. Average angular errors for (1) entirely real; (2) simulated polarisation ( $\phi$ and $\rho$ ); and (3) simulated intensity $(I)$ data were: $7.6^{\circ}, 6.4^{\circ}$ and $2.9^{\circ}$ respectively. This is rather striking as it shows the PS component has by far the largest associated error. This is presumably due to the resilience of polarisation phase data against reflectance properties compared to PS and the lesser need for carefully positioned light sources. It should be noted however, that such a breakdown of experimental error may change for certain target objects, such as where large areas of the surface have very low degree of polarisation.

These areas of low degree of polarisation have associated high noise levels and may be a potential weakness of the method. Results so far in this paper overcame this in a rather expensive manner by capturing 100 images in very rapid succession for each of the four polariser angles. The effects of using fewer images is illustrated by Fig. 10 which shows the estimated surface normal ( $y$ surface integrator is smoothing over the noise, and (2) the higher noise regions 

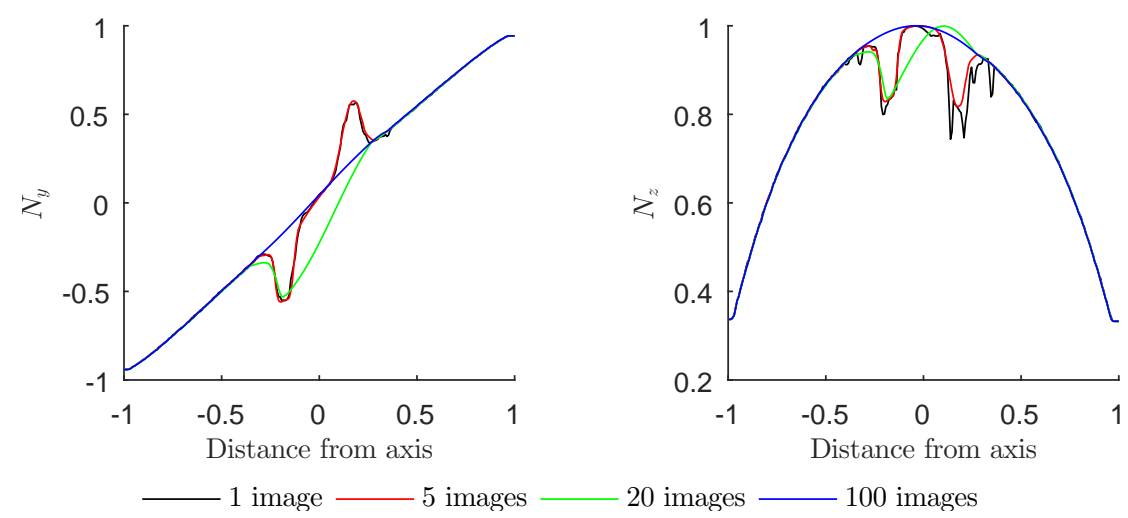

Figure 10: Comparison between surface normal estimates for varying number of images per polariser angle for a cross-section of the snooker ball.

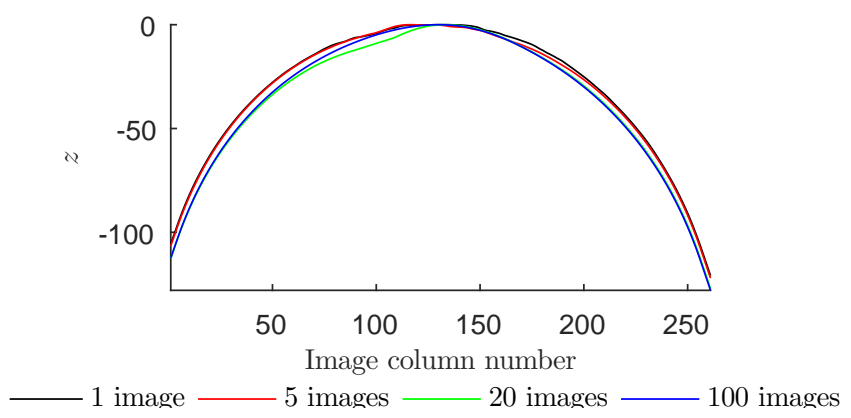

Figure 11: Comparison between surface depth estimates for varying number of images per polariser angle for a cross-section of the snooker ball.

correspond to areas of low zenith angle which have smaller effects on the integration than those of larger zenith angle.

\subsection{Other objects}

Surface normal estimates for two cylinders are shown in Fig. 12. For this case, the cylinders are both porcelain: one pure white and one multicoloured, as shown in Fig. 13. The results clearly show a close match to theory; with the main discrepancy being at the occluding contours. For the case shown, the axes of the cylinders were oriented parallel to the $x$-axis (see Fig. 1). Unfortunately, the method completely fails for the case where the cylinder axis is parallel to $y$-axis. This is due to the fact that all pixels on the surface in such cases appear 

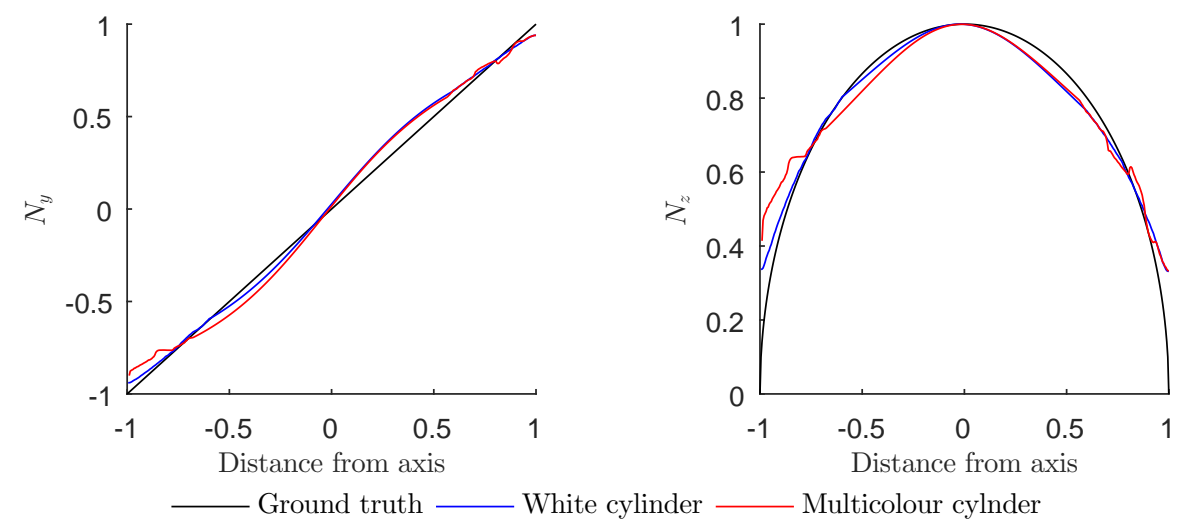

Figure 12: Comparison between surface normal estimates and ground truth for a white porcelain and multicoloured porcelain cylinder.

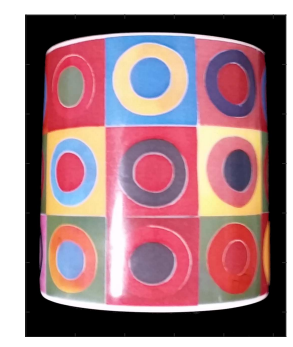

Figure 13: Photograph of the multicoloured cylinder used for Fig. 12

equally bright from each light source, making seed selection and region growing nearly impossible: a clear weakness of the method which will be addressed in future work.

Tests on objects of more complicated geometry are shown in Figs. 14 and 460 15. The general geometries of the fruits in Fig. 14 have clearly been reproduced, albeit with over-smoothing in places. The model dinosaur in Fig. 15 also has reasonable shape, despite a few areas of incorrect disambiguation on the foot and head. It is hoped that such issues might be resolved in future work by optimising the location and number of seed points used. 


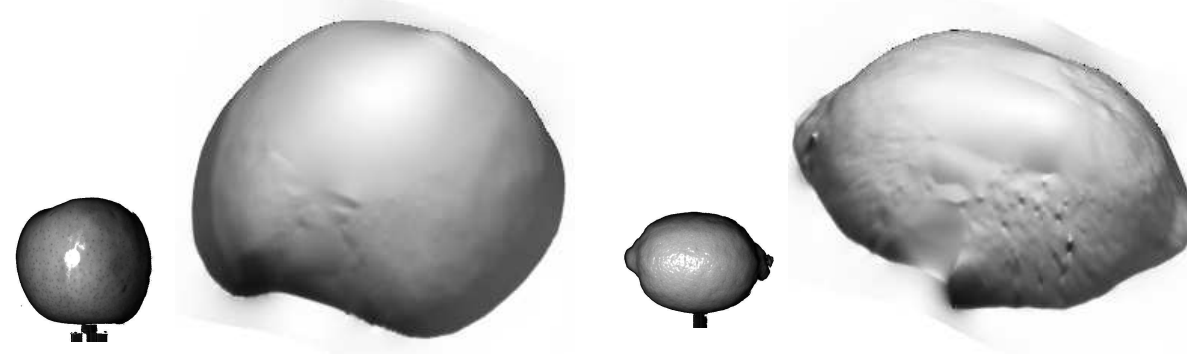

Figure 14: Surface reconstructions of an apple and lemon.

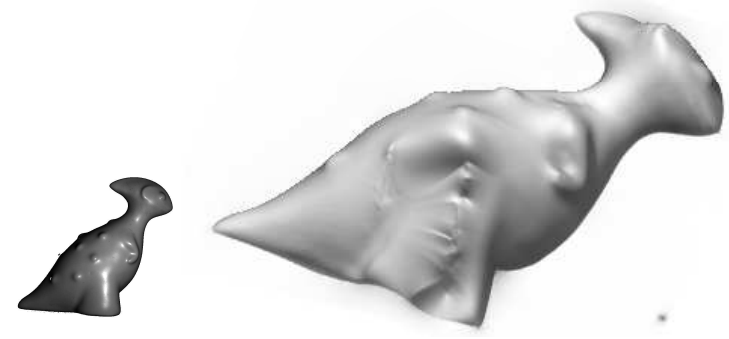

Figure 15: Surface reconstruction of a model dinosaur. 


\subsection{An alternative integrator}

The choice of the Frankot-Chellappa integration method for conversion from normals to depth was made since (1) it is well-established in the field and (2) it operates at high speed (much less than one second to reconstruct a VGA frame). However, the method is not very effective in certain circumstances. The most

figure, this significantly improves results for most of the surface ${ }^{1}$, The obvious disadvantage of this approach was that the integration took much longer $(\approx 40 \mathrm{~s}$ compared to $\approx 0.1 \mathrm{~s})$.

\section{Conclusion}

Results in this paper demonstrate the potential for use of polarisation information for scene understanding in the presence of both diffuse reflection

\footnotetext{
${ }^{1}$ The seemingly random pattern near the middle of the M-Estimator reconstruction is due to text printed on the surface.
} 

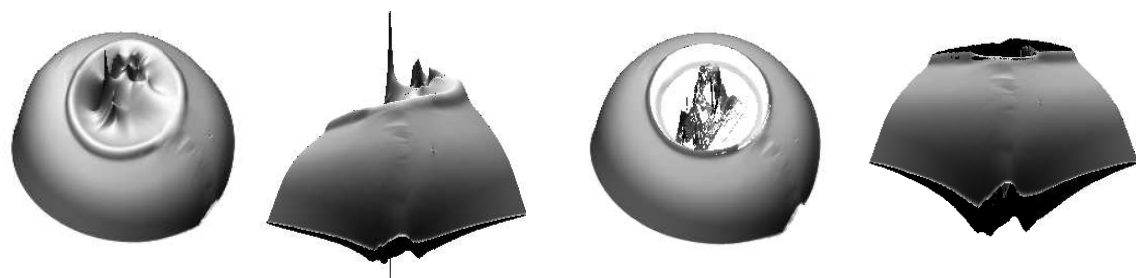

Figure 16: Reconstructions of the underside of a porcelain bowl, including the side view. The left reconstructions were based on the Frankot-Chellappa method, while those to the right use the M-Estimator.

and specular inter-reflections. The results show reconstruction quality that is improved or comparable with the baseline methods while needing only two illumination sources, which is competitive to many other methods in the field. At present, the data is captured using a rotating polariser and switching light sources meaning the data takes several seconds to capture. As stated in Section 2 however, the method can be applied to data captured from a polarisation camera such as the POLKA. This means that the only significant limitation in capture time relates to the light source switching, which has easily been shown to operate in the order of milliseconds $[5$. The processing time for this method is of the order five seconds at VGA resolution on an average personal computer.

The method has clearly demonstrated the use of polarisation information to reduce the number of light sources needed for photometric stereo to two. Unlike with many alternative methods in polarisation vision, this advantage does not come at the expense of needing to know a refractive index or making strong assumptions on reflectance properties. One major weakness of many PS methods is that of shadowing. While the novel method still suffers from reduced information at shadowed regions; only the zenith angle is affected. It is therefore predicted that the estimated azimuth angles for such regions may be combined with intensity information from one source only to fully recover shadowed surfaces in follow-on work. The dependence of the method on the reflectance properties of the surface is also deemed superior to many PS approaches since polarisation (at least phase angle) has little relationship to other 
reflectance parameters. That said, it is acknowledged that the proposed approach is still somewhat affected by reflectance properties due to the use of (8) for the $z$-component of the normal.

One limitation that should be addressed in future work relates to the open question of how to recover the geometry of an entire scene (as opposed to a single object). It may be that optimisation of seed point generation and weight-

${ }_{520}$ ing inter-object pixels in the integrator may reduce this limitation. Another limitation relates to the complications caused by very rough surfaces. The main problem caused by roughness for many polarisation-based methods is the depolarising effect of microscopic inter-reflections on the surface. This typically has a direct degrading effect on the reconstruction because the zenith angle is

525 inherently dependent on degree of polarisation. For the method proposed in this paper however, the zenith angle is entirely determined by PS, diminishing the effects of roughness. Nevertheless, the increased noise present due to lower degrees of polarisation from rough surfaces will still clearly impact results of the method to an extent.

\section{References}

[1] M. Z. Brown, G. D. Hager, Advances in computational stereo, IEEE Trans. Patt. Anal. Mach. Intell. 25 (2003) 993-1008.

[2] S. Foix, G. Alenyà, Lock-in time-of-flight (ToF) cameras: A survey, IEEE Sensors J. 11 (2011) 1917-1926.

[3] J. D. Durou, M. Falcone, M. Sagnona, Numerical methods for shape-fromshading: A new survey with benchmarks, Comp. Vis. Im. Understanding 109 (2008) 22-43.

[4] P. N. Belhumeur, D. J. Kriegman, A. L. Yuille, The bas-relief ambiguity, Intl. J. Comp. Vis. 35 (1999) 33-44.

${ }_{540}$ [5] M. F. Hansen, G. A. Atkinson, L. N. Smith, M. L. Smith, 3D face recon- 
structions from photometric stereo using near infrared and visible light, Comp. Vis. Im. Understanding 114 (2010) 942-951.

[6] W. Zhang, M. L. Smith, L. N. Smith, A. Farooq, Gender recognition from facial images: two or three dimensions?, J. Opt. Soc. Am. A 33 (2016) $333-344$.

[7] L. B. Wolff, T. E. Boult, Constraining object features using a polarisation reflectance model, IEEE Trans. Patt. Anal. Mach. Intell. 13 (1991) 635-657.

[8] E. Hecht, Optics, 3rd Edition, Addison Wesley Longman, 1998.

[9] M. Saito, Y. Sato, K. Ikeuchi, H. Kashiwagi, Measurement of surface orientations of transparent objects using polarization in highlight, in: Proc. CVPR, Vol. 1, 1999, pp. 381-386.

[10] D. Miyazaki, M. Kagesawa, K. Ikeuchi, Transparent surface modelling from a pair of polarization images, IEEE Trans. Patt. Anal. Mach. Intell. 26 (2004) 73-82.

[11] G. A. Atkinson, E. R. Hancock, Shape estimation using polarization and shading from two views, IEEE Trans. Patt. Anal. Mach. Intell. 29 (2007) 2001-2017.

[12] K. Berger, R. Voorhies, L. Matthies, Incorporating polarization in stereo vision-based 3D perception of non-Lambertian scenes, in: Proc. SPIE, Vol. 9837, 2016.

[13] V. Taamazyan, A. Kadambi, R. Raskar, Shape from mixed polarization, arXiv preprint: 1605.02066.

[14] D. Miyazaki, R. T. Tan, K. Hara, K. Ikeuchi, Polarization-based inverse rendering from a single view, in: Proc. ICCV, Vol. 2, 2003, pp. 982-987.

565 [15] O. Drbohlav, R. Šára, Unambiguous determination of shape from photometric stereo with unknown light sources, in: Proc. ICCV, 2001, pp. $581-586$. 
[16] O. Drbohlav, R. Šára, Specularities reduce ambiguity of uncalibrated photometric stereo, in: Proc. ECCV, 2002, pp. 46-62.

[17] G. A. Atkinson, E. R. Hancock, Two-dimensional BRDF estimation from polarisation, Comp. Vis. Im. Understanding 111 (2008) 126-141.

[18] T. Ngo, H. Nagahara, R. Taniguchi, Shape and light directions from shading and polarization, in: In Proc. CVPR, 2015, pp. 2310-2318.

[19] N. M. Garcia, I. de Erausquin, C. Edmiston, V. Gruev, Surface normal reconstruction using circularly polarized light, Opt. Express 23 (2015) 1439114406.

[20] O. Morel, C. Stolz, F. Meriaudeau, P. Gorria, Active lighting applied to three-dimensional reconstruction of specular metallic surfaces by polarization imaging, Applied Optics 45 (2006) 4062-4068.

[21] W. Smith, R. Ramamoorthi, S. Tozza, Linear depth estimation from an uncalibrated, monocular polarisation image, in: In Proc. ECCV, 2016, pp. $109-125$.

[22] S. Rahmann, N. Canterakis, Reconstruction of specular surfaces using polarization imaging, in: Proc. CVPR, 2001, pp. 149-155.

[23] S. Rahmann, Reconstruction of quadrics from two polarization views, in: Proc. IbPRIA, 2003, pp. 810-820.

[24] C. P. Huynh, A. Robles-Kelly, E. Hancock, Shape and refractive index recovery from single-view polarisation images, in: Proc. CVPR, 2010, pp. $1229-1236$.

[25] A. Kadambi, V. Taamazyan, B. Shi, R. Raskar, Polarized 3D: High-quality depth sensing with polarization cues, in: Proc. ICCV, 2015.

[26] G. A. Atkinson, E. R. Hancock, Recovery of surface orientation from diffuse polarization, IEEE Trans. Im. Proc. 15 (2006) 1653-1664. 
[27] L. B. Wolff, Diffuse-reflectance model for smooth dielectric surfaces, J. Opt. Soc. Am. A 11 (1994) 2956-2968.

[28] Fraunhofer Institute for Integrated Circuits, http:// WWW.iis.fraunhofer.de/en/ff/bsy/tech/kameratechnik/ polarisationskamera.html, [Accessed: 1 March 2017].

[29] W. Burger, M. J. Burge, Principles of Digital Image Processing: Fundamental Techniques, Springer, 2010.

[30] N. Karpel, Y. Schechner, Portable polarimetric underwater imaging system with a linear response, in: Proc. SPIE, Vol. 5432, 2004.

[31] R. J. Woodham, Photometric method for determining surface orientation from multiple images, Optical Engineering 19 (1980) 139-144.

[32] R. F. Saracchini, J. Stolfi, H. C. G. Leitão, G. A. Atkinson, M. L. Smith, A robust multi-scale integration method to obtain the depth from gradient maps, Comp. Vis. Im. Understanding 116 (2012) 882-895.

[33] R. T. Frankot, R. Chellappa, A method for enforcing integrability in shape from shading algorithms, IEEE Trans. Patt. Anal. Mach. Intell. 10 (1988) 439-451.

[34] A. Agrawal, R. Raskar, R. Chellappa, What is the range of surface reconstructions from a gradient field?, in: Proc. ECCV, 2006, pp. 578-591. 
LaTeX Souce Files
Click here to download LaTeX Souce Files: sourceFiles.zip

LaTeX Souce Files
Click here to download LaTeX Souce Files: sourceFiles.zip Click here to download LaTeX Souce Files: sourceFiles.zip

pros

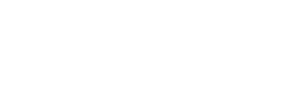

(1)

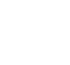

